\title{
Surgical management of pulmonary endarterectomy avoiding deep hypothermia: the Pavia experience
}

\author{
Andrea M. D'Armini ${ }^{1,2}$, Anna Celentano ${ }^{1}$, Alessia Alloni ${ }^{3}$, Giuseppe Silvaggio ${ }^{3}$, Cristian Monterosso ${ }^{3}$, \\ Carlo Pellegrini ${ }^{1,3}$, Stefano Ghio ${ }^{4}$ \\ ${ }^{1}$ Department of Clinical, Surgical, Pediatric and Diagnostic Sciences, University of Pavia School of Medicine, Italy; ${ }^{2}$ Division of Cardiac Surgery \\ 2 and Pulmonary Hypertension Center, Foundation I.R.C.C.S. Policlinico San Matteo, Pavia, Italy; ${ }^{3}$ Division of Cardiac Surgery 1, Foundation \\ I.R.C.C.S. Policlinico San Matteo, Pavia, Italy; ${ }^{4}$ Division of Cardiology, Foundation I.R.C.C.S. Policlinico San Matteo, Pavia, Italy \\ Correspondence to: Andrea M. D’Armini, MD. Division of Cardiac Surgery 2 and Pulmonary Hypertension Center, Foundation I.R.C.C.S. Policlinico \\ San Matteo, Viale Golgi 19, 27100 Pavia, Italy. Email: andreamaria.darmini@unipv.it; a.darmini@smatteo.pv.it.
}

Submitted Nov 16, 2021. Accepted for publication Dec 17, 2021.

doi: 10.21037/acs-2021-pte-22

View this article at: https://dx.doi.org/10.21037/acs-2021-pte-22

Chronic thromboembolic pulmonary hypertension (CTEPH) is a rare disease included in group 4 of the pulmonary hypertension (PH) clinical classification system (1). CTEPH is characterized by the presence of obstructed pulmonary arteries by thromboembolic material and the remodeling of the remaining patent vessels (2). These vascular changes lead to $\mathrm{PH}$, decreased right ventricular function and subsequent right heart failure, clinical deterioration and premature mortality (3). Although the actual incidence of CTEPH is unknown, a cumulative incidence of $0.1-9.1 \%$ within the first two years after a symptomatic pulmonary embolism event, has been reported (2).

Pulmonary endarterectomy (PEA) is the gold standard treatment for patients with CTEPH. In expert centers, it is a low-risk procedure with in-hospital mortality rates $<5 \%$, closely associated with the surgeon's experience $(3,4)$. Additionally, the long-term results are excellent. Survival rates of $86 \%$ at 1 -year, $79 \%$ at 5 -year and $72 \%$ at 10 -year have been reported and are significantly better than those who have not undergone PEA (4). PEA is curative in most cases, with normalization of pulmonary pressures and clinical improvement (5).

The surgical technique of PEA, which was introduced by the San Diego group more than forty years ago, has changed slightly over the years $(5,6)$. Our center has progressively modified the original San Diego surgical technique starting in 2003 , with the use of moderate hypothermia $\left(24^{\circ} \mathrm{C}\right)$ instead of deep hypothermia $\left(18{ }^{\circ} \mathrm{C}\right)$ and intermittent short periods of circulatory arrest $(\approx 7-10$ minutes) followed by short periods of reperfusion ( $\approx 5-7$ minutes) instead of a single period of circulatory arrest (20 minutes) for each lung (with a maximum of a third period of circulatory arrest in complicated and challenging cases). Other differences in the technique we currently use are avoidance of aortic clamping and cardioplegia infusion. Myocardial protection is obtained in addition to moderate hypothermia by ventricular fibrillation and left ventricle venting. Routinely, we also use NIRS (near infrared spectroscopy) to check cerebral oxygenation during both circulatory arrest and reperfusion periods, with the possibility of tailoring the duration of each period. These modifications give us the possibility of a safe, longer cumulative circulatory arrest time (CCAT). In our hands, longer CCAT allows us to be more accurate and precise in the treatment of chronic thromboembolic material, since we can reach even the most distal lesions and explore the majority of the pulmonary arteries even in those without apparent chronic thromboembolic material at computed tomography (CT) scan. Another advantage of multiple repeated reperfusion periods is to avoid ischemic phenomena in all organs, especially in the abdomen.

We found that the new protocol was associated with an improvement in postoperative respiratory function, a reduction in days of mechanical ventilation, a reduction of postoperative infections and a significant improvement in post-operative courses without adverse events. We also demonstrated an improvement in hemodynamic and functional results (7). PEA with 
repeated short periods of circulatory arrest, moderate hypothermia and longer CCAT did not result in any neuropsychological complications and may even lead to post-surgical psychological improvements (8). This technique, with more safe time in our hands, has allowed us to perform a more accurate dissection of all branches, even in case of distal lesions (9). Another advantage of these repeated periods of reperfusion could be, especially in the elderly patients where the whole microvascular diseases are more frequent, to avoid dangerous prolonged periods of ischemia (10).

Since April 1994 to October 2021, 1,108 patients underwent PEA at our center. More than 850 of them (first case October 2009 case \#245) were treated using our modified protocol (Pavia technique for PEA). In these patients the mean CCAT was $93 \pm 27$ minutes. We have also started to count the number of treated branches (NTB) (first case February 2010 case \#271). The thromboembolic material is reconstructed on the bench, photographed and the treated branches counted by two different operators. In these patients the mean NTB was $40 \pm 16$. The CCAT was correlated with the NTB: more NTB, longer CCAT.

We have observed a strong correlation between the NTB and the hemodynamic and functional results. This correlation (that is, the greater the number of treated branches the better the hemodynamic results) was statistically evident already at the three-month follow-up control and was maintained at the twelve-month followup control. This data shows that the treatment of multiple branches during PEA, even with a longer period of CCAT, does not impact the risk of PEA. Our mortality rate is comparable to the mortality rate in the International Registry, when adjusted for the WHO functional class (3).

Between January 2020 and October 2021, in the months with the highest peak of Covid-19, restrictions in Italy allowed us to perform PEA only in advanced patients with functional class III and IV. Throughout this period, we performed 131 PEA with a functional class distribution of 16 patients in WHO functional class II (12.2\%), 65 patients in WHO functional class III (49.6\%) and 50 patients in WHO functional class IV (38.2\%) with an in-hospital mortality of $2.3 \%$ (three deaths).

In conclusion, these results support our decision to use moderate hypothermia with short periods of circulatory arrest, alternating to short periods of reperfusion. This technique allows more safe time to explore the pulmonary arteries and to remove even the most distal lesions. This technique guarantees patients a safe protocol and excellent results.

\section{Acknowledgments}

Funding: None.

\section{Footnote}

Conflicts of Interest: The authors have no conflicts of interest to declare.

Open Access Statement: This is an Open Access article distributed in accordance with the Creative Commons Attribution-NonCommercial-NoDerivs 4.0 International License (CC BY-NC-ND 4.0), which permits the noncommercial replication and distribution of the article with the strict proviso that no changes or edits are made and the original work is properly cited (including links to both the formal publication through the relevant DOI and the license). See: https://creativecommons.org/licenses/by-nc-nd/4.0/.

\section{References}

1. Galiè N, Humbert M, Vachiery JL, et al. 2015 ESC/ERS Guidelines for the diagnosis and treatment of pulmonary hypertension: The Joint Task Force for the Diagnosis and Treatment of Pulmonary Hypertension of the European Society of Cardiology (ESC) and the European Respiratory Society (ERS): Endorsed by: Association for European Paediatric and Congenital Cardiology (AEPC), International Society for Heart and Lung Transplantation (ISHLT). Eur Heart J 2016;37:67-119.

2. Lang IM, Pesavento R, Bonderman D, et al. Risk factors and basic mechanisms of chronic thromboembolic pulmonary hypertension: a current understanding. Eur Respir J 2013;41:462-8.

3. Delcroix M, Lang I, Pepke-Zaba J, et al. Long-Term Outcome of Patients With Chronic Thromboembolic Pulmonary Hypertension: Results From an International Prospective Registry. Circulation 2016;133:859-71.

4. Jenkins D, Madani M, Fadel E, et al. Pulmonary endarterectomy in the management of chronic thromboembolic pulmonary hypertension. Eur Respir Rev 2017;26:160111.

5. Jamieson SW, Kapelanski DP. Pulmonary endarterectomy. Curr Probl Surg 2000;37:165-252.

6. Mayer E, Klepetko W. Techniques and outcomes of pulmonary endarterectomy for chronic thromboembolic pulmonary hypertension. Proc Am Thorac Soc 2006;3:589-93. 
7. Morsolini M, Nicolardi S, Milanesi E, et al. Evolving surgical techniques for pulmonary endarterectomy according to the changing features of chronic thromboembolic pulmonary hypertension patients during 17-year single-center experience. J Thorac Cardiovasc Surg 2012;144:100-7.

8. Vanini B, Grazioli V, Sciortino A, et al. Neuropsychological outcomes after pulmonary endarterectomy using moderate hypothermia and periodic circulatory arrest. J Heart Lung

Cite this article as: D'Armini AM, Celentano A, Alloni A, Silvaggio G, Monterosso C, Pellegrini C, Ghio S. Surgical management of pulmonary endarterectomy avoiding deep hypothermia: the Pavia experience. Ann Cardiothorac Surg 2022;11(2):177-179. doi: 10.21037/acs-2021-pte-22
Transplant 2018;37:860-4.

9. D'Armini AM, Morsolini M, Mattiucci G, et al. Pulmonary endarterectomy for distal chronic thromboembolic pulmonary hypertension. J Thorac Cardiovasc Surg 2014;148:1005-11; 1012.e1-2; discussion 1011-2.

10. Grazioli V, Ghio S, Pin M, et al. Pulmonary endarterectomy in the octogenarian population: safety and outcomes. J Cardiovasc Med (Hagerstown) 2021;22:567-71. 